\title{
Hydraulic characterization of Diesel and water emulsions using momentum flux
}

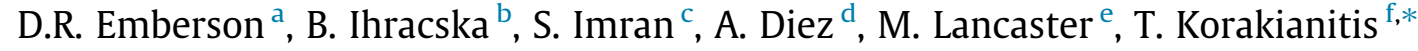 \\ ${ }^{a}$ School of Engineering and Materials Science, Queen Mary University of London, Mile End Road, E1 4NS, UK \\ ${ }^{\mathrm{b}}$ School of Engineering, University of Hertfordshire, AL10 9AB, UK \\ c Department of Mechanical Engineering, University of Engineering and Technology (City Campus), Lahore, Pakistan \\ ${ }^{\mathrm{d}}$ Izmir Institute of Technology, Urla, Izmir 35430, Turkey \\ ${ }^{\mathrm{e}}$ Advanced Engine Research Ltd, Basildon, Essex SS13 1LE, UK \\ ${ }^{\mathrm{f}}$ Parks College of Engineering, Aviation and Technology, Saint Louis University, St. Louis, MO 63103, USA
}

\section{H I G H L I G H T S}

- Fuel injection momentum flux was measured using a force transducer.

- Diesel fuel and Diesel fuel and water emulsions containing $10 \%$ and $20 \%$ water examined.

- The mass flow, discharge coefficient, injection velocity and momentum efficiency were evaluated.

- Emulsion sprays had a lower discharge coefficient and higher injection velocity than the Diesel.

- The emulsified fuels had a larger momentum efficiency.

\section{A R T I C L E I N F O}

\section{Article history:}

Received 29 May 2015

Received in revised form 5 August 2015

Accepted 5 August 2015

Available online 17 August 2015

\section{Keywords:}

Diesel water emulsions

Fuel injection

Momentum flux

Instantaneous mass flow rate

\begin{abstract}
A B S T R A C T
Diesel and water emulsions have the potential to be used in compression ignition engines to control the emissions of $\mathrm{NO}_{x}$ and PM. Very little is known about the influence emulsification will have on the fuel sprays formed during injection. This paper outlines the measurement of the momentum flux of injection sprays of Diesel fuel and Diesel fuel emulsions containing $10 \%$ and $20 \%$ water, with the goal of hydraulically characterizing the sprays and identifying the influence emulsification may have on them. The momentum flux, mass flow, instantaneous mass flow, discharge coefficient, injection velocity, momentum coefficient and momentum efficiency have been examined. The injections were carried out in a high pressure chamber filled with nitrogen. The measured momentum flux is observed to increase with increasing injection pressure in a linear form. Increasing the ambient density in the chamber resulted in a decrease in the measured momentum flux. The emulsified fuel sprays had a very similar momentum flux as the neat Diesel fuel sprays. The total mass of emulsified fuel injected was less than for neat Diesel at corresponding condition. The instantaneous mass flow rate was determined using a normalized form of the momentum flux measurement and the independently measured total mass injected. The emulsions tended to have a lower discharge coefficient and there is no evidence that the nozzle is cavitating at these conditions. The emulsified fuels have tended to have a higher injection velocity than the neat Diesel fuel sprays. The momentum efficiency is introduced, which uses the instantaneous mass measurement and the theoretical velocity of the spray. The emulsified fuels have a larger momentum efficiency, a result of their high injection velocity compared with the neat Diesel fuel.
\end{abstract}

(C) 2015 Published by Elsevier Ltd.

\section{Introduction}

There is currently a heavy reliance on fossil derived fuel. It is accepted that there is a need to utilize these fuels as efficiently

\footnotetext{
* Corresponding author. Tel.: +1 3149778231.

E-mail address: korakianitis@alum.mit.edu (T. Korakianitis).
}

as possible alongside examining possible alternatives to replace and extend their use. Much of the dependence on these fossil derived fuels stems from their use in the internal combustion engine (ICE), an established technology that is used extensively throughout the world. There is currently no alternative that is as flexible and efficient. 


\section{Nomenclature}

$\begin{array}{ll}\text { Symbols } & \\ m_{a v} & \text { average mass flow rate } \\ \dot{m} & \text { instantaneous mass flow rate } \\ \dot{M} & \text { momentum flux } \\ \eta_{m} & \text { momentum efficiency } \\ \rho_{f} & \text { fuel density } \\ \rho & \text { density } \\ A_{c} & \text { contracted area } \\ A_{g e o} & \text { geometrical cross sectional area } \\ C_{a} & \text { area contraction coefficient } \\ C_{c} & \text { contraction coefficient } \\ C_{d} & \text { discharge coefficient } \\ C_{v} & \text { velocity coefficient } \\ D_{i} & \text { internal diameter } \\ D_{o} & \text { outer diameter } \\ d A & \text { area differential } \\ d V & \text { volume differential } \\ F & \text { force } \\ \mathrm{HC} & \text { hydrocarbon }\end{array}$

$\begin{array}{ll}K & \text { cavitation number } \\ P & \text { pressure } \\ R e & \text { Reynolds number } \\ v_{\text {eff }} & \text { effective velocity } \\ v_{B} & \text { theoretical velocity } \\ v & \text { velocity } \\ \mathrm{NO}_{x} & \text { nitrogen oxides } \\ \mathrm{PM} & \text { particulate matter } \\ t & \text { time }\end{array}$

Abbreviations

$\mathrm{CI} \quad$ compression ignition

EOI end of injection

HLB hydrophilic-lipophilic balance

ICE internal combustion engine

SEM scanning electron microscope

SOI start of injection

TTL transistor transistor logic

The use of the ICE is accompanied by the emission of a range of combustion products into the atmosphere. The legislation of these emissions has become more stringent as understanding and concern about environmental pollution has drastically increased. Of particular concern are the emissions of soot, particulate matter (PM) and nitrogen oxides $\left(\mathrm{NO}_{x}\right)$. This paper focuses a fuel additive in the form of water to create fuel and water emulsions. Such emulsions have shown the potential to assist in the control of these emissions from a compression ignition engine [1].

\subsection{Emulsions}

A water in fuel emulsion is a mixture of a base fuel, usually defined as the oil phase or continues phase, with water, the dispersed phase. The two liquids are immiscible, hence the mixture is thermodynamically unstable. The stability of the mixture is increased with the addition of a surfactant. A surfactant is a substance that is adsorbed onto the surfaces or interfaces of the system and alters the surface or interfacial free energies of those surfaces (or interfaces). An interface indicates a boundary between any two immiscible phases, a surface is an interface where one phase is a gas, usually air [2].

Water is added to the base fuel in the range of $10-30 \%$ by mass or volume. A small amount of surfactant, usually in the range 1-5\% is used to stabilize the emulsion. The emulsion is then utilized in the $\mathrm{CI}$ engine as normal. The use of emulsified fuels has been shown to reduce $\mathrm{NO}_{x}, \mathrm{CO}$, soot, hydrocarbons (HC) and PM emissions when used in a $\mathrm{CI}$ engine $[1,3]$. Water in fuel emulsions have also been shown to slightly improve the brake thermal efficiency of a CI engine $[4,5]$.

Emulsified fuels usually exhibit an increase in viscosity (due to the dispersion of the water droplets) with respect to the base oil phase [6]. This in turn will have an effect on spray dynamics. Other spray dynamic effects of emulsification include the longer liquid penetration which can be attributed to the low volatility of the water [7]. It is postulated that spray momentum [6] and velocity is altered compared with the base fuel, leading the flame lift of length to increase, allowing more air to be entrained before the combustion, leading to leaner mixtures within the fuel jet during the quasi-steady combustion. As a consequence, in-cylinder soot formation could be reduced [8]. There has been very little work done investigating the spray characteristics of emulsified fuels which contributes to a general lack of understanding concerning their use in direct injection $\mathrm{CI}$ engines. This study goes some way to address this through the hydraulic characterization of emulsified Diesel fuel.

\subsection{Hydraulic characterization of fuel sprays}

The most basic hydraulic characterization of a fuel injection is the measurement of the fuel mass injected $(m)$. An average mass flow rate $m_{a v}$ may be determined from this mass flow through the nozzle and the time duration of the injection. The mass flow rate is crucial in gaining a more complete understanding of the spray and combustion process in a $\mathrm{CI}$ or a direct injection, gasoline, spark ignition (GDI) engine. When applied to fuel metering accuracy and injection rate control, mass flow rate is undoubtedly a key parameter in controlling the spray formation, evolution and hence combustion. The instantaneous mass flow rate, $\dot{m}$, a time resolved measurement of the mass flow rate is much more useful.

A number of methods are employed to measure the injection mass flow rate. The two main methods used to measure $\dot{m}$ are based upon the Zeuch method and the Bosch method [9]. With the Bosch method, a pressure wave is generated by the injection of a volume of fuel into a length of tubing containing a compressible fluid, usually Diesel fuel. This pressure variation is measured and scaled to determine $\dot{m}$.

For the Zeuch method, injection is conducted in a closed, fixed volume chamber filled with the injection fluid, the resultant pressure rise in the chamber is recorded from which $\dot{m}$ is determined.

Nabers and Siebers [10] applied an alternative method which relies on independently measuring the momentum flux of the fuel spray emanating from the nozzle orifice and the total mass of fuel injected during the injection event. The technique has been applied in a number of other works [11-14]. Spray momentum flux is measured indirectly through the measurement of an impact force of the spray. This is achieved by placing a linear force transducer in front of the nozzle orifice, very close to the exit, with the transducer's measuring surface normal to the spray central axis. The control volume, $\mathrm{CV}$, into which the spray is forming is considered in Fig. 1, with the spray impinging on a target, which is directly coupled to the force transducer's measuring surface. The spray target interaction is governed by the following momentum conservation equation 


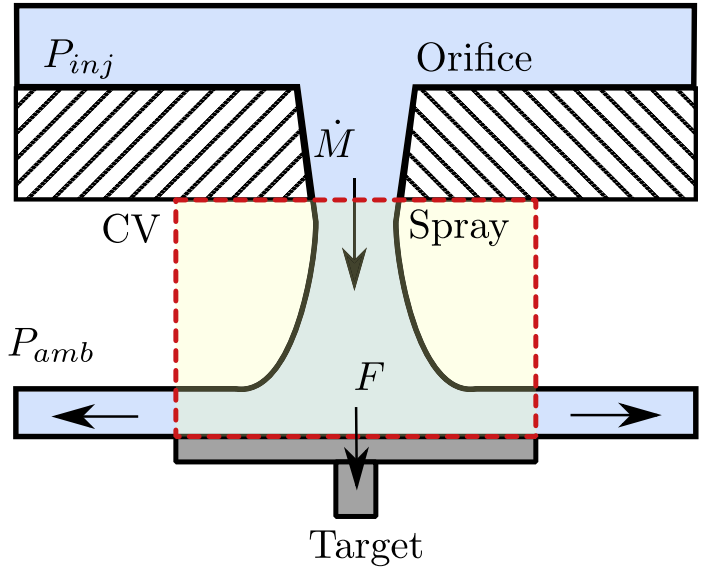

Fig. 1. Conceptual control volume of fuel injection spray emerging from the injector nozzle and striking a target attached to a force transducer's measuring sensor. $\mathrm{CV}=$ control volume, $P_{a m b}=$ ambient pressure, $P_{i n j}=$ injection pressure, $F=$ force measured by transducer, $\dot{M}=$ fuel momentum flux.

$F=\frac{\partial}{\partial t} \int_{C V} \rho v d V+\int_{A} v^{2} \rho d A$

where $v$ and $\rho$ is the velocity and density in the control volume, $d V$ is the volume differential, $d A$ is differential area taken as a vector normal to the control surface of the control volume and $F$ is the resultant force acting in the control volume. The first term on the right hand side is the rate of change of momentum stored in the control volume and the second term is the momentum flux through the control volume. The equation is considered along the spray axis and simplified assuming: the liquid jet area exiting the orifice is equal to the geometrical orifice area, $A_{\text {geo }}$; there is no air entrainment along the spray axis, hence air velocity is zero; there are negligible effects due to gravity; there is negligible mass of fluid in the control volume that is accelerating which makes the first term of Eq. (1) zero; spray deviation at the target face is orthogonal to the spray central axis so the fluid exiting the control volume has no axial velocity component; density is the density of the liquid $\rho_{f}$ which is constant and; the liquid jet exiting the orifice has a uniform velocity $v_{\text {eff }}$, (effective velocity) [15]. The assumption that the liquid jet area exiting the orifice is equal to the geometrical orifice area, $A_{\text {geo }}$ is reliant on there being no cavitation present.

The resultant force $F$ experienced by the transducer target is given by the second term of Eq. (1) with the axial velocity of the liquid jet, $v_{\text {eff }}$. The force equates to the injected momentum flux at the orifice exit, $F=\dot{M}$. The interaction experienced by the target is now expressed in the form

$\dot{M}=\rho_{f} A_{g e o} v_{e f f}^{2}$

From Eq. (2), we see that the velocity of liquid jet, $v_{\text {eff }}$, is proportional to the root of the momentum flux, $v_{\text {eff }} \propto \sqrt{\dot{M}}$. The instantaneous mass flow rate $\dot{m}$ is given by

$\dot{m}=\rho_{f} A_{\text {geo }} v_{\text {eff }}$

It follows that the instantaneous mass flow rate is proportional to the square root of the momentum flux $\dot{m}=\propto \sqrt{\dot{M}}$. Naber and Siebers [10] suggest that the relationship between the instantaneous mass flow rate and the momentum flux can be determined by normalizing the square root of the transducers output by the area under the curve for the square root of the output, $\left(\int_{0}^{t} \sqrt{\dot{M}} \cdot d t\right)$. The normalization results in a profile proportional to the injection velocity which when multiplied by the total mass per injection event gives the instantaneous mass flow rate. The normalization is conducted using Eqs. (2) and (3) to give the mass flow rate in the form [16]

$\dot{m}=\sqrt{\dot{M} \rho_{f} A_{g e o}}$

with the total mass injected during the injection event given by

$m=\int_{0}^{t} \dot{m} \cdot d t=\int_{0}^{t} \sqrt{\dot{M} \rho_{f} A_{g e o}} \cdot d t$

to give the instantaneous mass flow rate by

$\dot{m}=\frac{\sqrt{\dot{M} \rho_{f} A_{g e o}}}{\int_{0}^{t} \sqrt{\dot{M} \rho_{f} A_{g e o}} \cdot d t} m=\frac{\sqrt{\dot{M}}}{\int_{0}^{t} \sqrt{\dot{M}} \cdot d t} m$

Fuel density and orifice area drop out of the normalization and the instantaneous mass flow rate may be determined using the momentum flux (the transducer output) and the total mass of fuel injected during the injection event. The total mass injected can be independently measured by collecting the injected fuel in a container and weighing the mass from a number of injections. This method has been employed in the works $[10,16,17]$.

The discharge coefficient is defined as the ratio of the actual mass flow rate through an orifice to the theoretical mass flow through the orifice, for the same fluid and same pressure drop. It may be thought of as the efficiency of the nozzle (on a mass flow basis) which characterizes the mass flux through the orifice and may be determined using

$C_{d}=\frac{\dot{m}}{\dot{m}_{\text {th }}}=\frac{\dot{m}}{A_{\text {geo }} \rho_{f} v_{B}}=\frac{\dot{m}}{A_{\text {geo }} \sqrt{\rho_{f} 2 \Delta P}}$

The discharge coefficient may be divided into two parts. The flow velocity may be reduced from the theoretical maximum, $v_{B}$, to the an effective velocity, $v_{\text {eff }}$, due to friction, boundary layer effects and turbulence in the orifice. This reduction in velocity is accounted for by the velocity coefficient, $C_{v}$, defined as the ratio of the effective velocity to the theoretical maximum. The second part of the discharge coefficient is accounted for by the area contraction coefficient, $C_{a}$. The area contraction coefficient accounts for flow area losses as a result of vapor bubbles generated by cavitation, non-uniform velocity profiles at the outlet section hole and changes in fluid density [15]. Momentum flux measurements have previously been employed to examine the $C_{d}, C_{v}$ and $C_{a}$ of nozzles [15].

The cavitation number appears in the literature in a number of forms but is usually based upon the pressure difference across the injector orifice [18]. A common form is that based on the work of Nurick [19] defining the cavitation number as

$K=\frac{P_{i n j}-P_{v}}{P_{i n j}-P_{a m b}}=\frac{P_{i n j}-P_{v}}{\Delta P}$

At the onset of cavitation the discharge coefficient decreases. In previous works the vapor pressure has been omitted due to its extremely small size compared with the other pressures [15]. A cavitating flow through an orifice will reduce the area cross section, reducing the discharge coefficient. To simplify any analysis the vapor region is treated as a fixed, slip boundary which occupies a fixed fraction of the orifice section. This is most likely to occur at the throat, near the inlet to the orifice as this is where the liquid undergoes its largest direction change and hence velocity change.

The discharge coefficient becomes proportional to the square root of the cavitation parameter [19] and is expressed as

$C_{d}=C_{c} \sqrt{K}$ 
$K$ increases as injection pressure decreases or back pressure increases; with an increase in $K$ there is a moment where the cavitation will disappear and the discharge coefficient will become constant, this is termed $K_{\text {crit }}$. For values above $K_{\text {crit }}$ the flow will be pure liquid phase and will be dependent on the Reynolds number [19]. The plot of the measured discharge coefficient using the momentum flux and the square root of the cavitation number is used to examine the cavitation of a nozzle and identify the injection pressure or fluid conditions where cavitation initiates $[13,15,18]$.

\subsection{Scope of this study}

The work presented in this study applies the measurement of the momentum flux technique to the injection sprays of Diesel fuel and two emulsified Diesel fuels containing $10 \%$ and $20 \%$ water by mass, D10 and D20 respectively. The technique has been applied to hydraulically characterize the injections using the momentum flux, mass flow, instantaneous mass flow, discharge coefficient and injection velocity. The dimensionless parameters, the momentum coefficient and momentum efficiency are introduced as alternative methods to characterize the injections.

\section{Experimental set-up}

\subsection{Momentum flux measurement device}

A piezoelectric force transducer with a range of at least $0-10 \mathrm{~N}$ was required to measure the spray impact force. The short time scale of the measurement required a device with a rise time of the order of a few microseconds $(<10 \mu \mathrm{s})$. The short time scale and the oscillatory nature of the measurement required a device with a high natural frequency of at least $50 \mathrm{kHz}$ [17]. The transducer sensing face which is impacted by the high pressure fuel jet needed to be resistant to physical and chemical erosion. The Kistler model 9215 force transducer was chosen for this study and has been used in previous work including [16,17,20-23]. The force transducer is a pre-loaded, piezoelectric force transducer used for measuring quasi-static and dynamic forces from -20 to $200 \mathrm{~N}$ (tension and compression) with the ability to measure loads as small as $1 \mathrm{mN}$ and a natural frequency in excess of $50 \mathrm{kHz}$. The transducer design consists of the sensor body with on outer thread for mounting. The face of the transducer consists of a $2 \mathrm{~mm}$ threaded bore to accept a screw. It is this screw which introduced the force into the transducer's sensing component. In original form this screw's head is dome shape. This screw was replaced with a stainless steel screw with the head machined to a smooth surface, $4 \mathrm{~mm}$ in diameter. This screw was the spray target and is referred to as the target from here.

All injections took place in the high pressure chamber as detailed in [24]. The chamber was charged with nitrogen at the ambient temperature of $20^{\circ} \mathrm{C}$. Two chamber pressures were considered, 20 and $30 \mathrm{bar}$, corresponding to two ambient gas densities of $\rho_{a}=22.6 \mathrm{~kg} / \mathrm{m}^{3} \rho_{a}=34.5 \mathrm{~kg} / \mathrm{m}^{3}$ respectively. The transducer target was positioned in front of the nozzle orifice by an aluminum bracket attached to a supporting bar which in turn was attached to the base of the high pressure chamber (see Fig. 2). The central axis of the orifice, relative to the central axis of the injector body, was measured using a silicone mold of the nozzle orifice, a measurement technique used previously in [15].

The bracket design allowed the transducer target distance from the nozzle to be adjusted. For all tests here the distance was kept to a minimum, due to nozzle geometry this was found to be $0.5 \mathrm{~mm}$. The distance was measured using a feeler gauge which was used to position the target at this distance. Other distances have been used in previous works in the range $0.5-10 \mathrm{~mm}[11,12,14-16,25]$. The work [14] found very little difference in the momentum flux

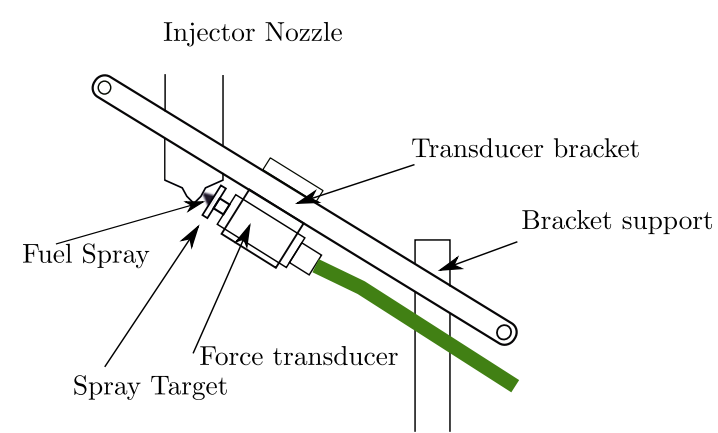

Fig. 2. Schematic diagram of the force transducer fitted with the target presented to an emerging spray.

measured for the distance range used but examination of their figures suggest the lower value of $0.5 \mathrm{~mm}$ may have provided the better result. Once fixed in position the transducer and bracket were not moved in any way during the data collection period. Good alignment was desired and strived for but small deviations in the angle away from perfect alignment are inevitable. This error will be small as the measured force is proportional to $\cos (\Delta \theta)$ where $\theta$ is the deviation away from perfect alignment, for small angles, $\cos \theta$ is approximately 1 .

The signal from the transducer was amplified by a Kistler 5007 charge amplifier. The voltage output signal from the charge amplifier was acquired at a frequency of $100 \mathrm{kHz}$ by a 6024E DAQ card (National Instruments). The injection trigger was handled by the custom LabView program which also handled the data acquisition. The data recorded consisted of a voltage signal which was linearly proportional to the force exerted on the target by the spray.

The fuel injection system was a research common rail system using a compressed air driven high pressure pump (Sprague P4333) and a stock common rail fitted with a piezo force transducer. The injector used was a Siemens, micro-sac, piezo type with 7 holes, $120 \mu \mathrm{m}$ in diameter and is controlled by a Hartridge driver. The use of a piezo actuated injector results in a very rapid opening and closing force applied to the needle, with a reduced transient period in the measured momentum flux. This has a positive effect on the spray-target interaction at the start of injection, with the time it takes for the spray to reach full orthogonal deviation at the target minimized. For the purpose of this study, all but one hole was laser welded closed to avoid spray interference with the body and bracket of the force transducer. The $K$-factor (nozzle conicity) is given by $K=\frac{D_{i}-D_{o}}{10}$. The nozzle $K$-factor was determined using the measurements made in an SEM of the silicone mold of the nozzle geometry, shown in Table 1 . The inlet radius was also measured in the SEM and was determined to be approximately $70 \mu \mathrm{m}$.

Data recording started with the injection TTL trigger which was sent to a Hartridge driver. Injection duration for all test was set to $0.5 \mathrm{~ms}$ (TTL on time of $0.5 \mathrm{~ms}$ ). Three injection pressures were considered, 500, 700 and 1000 bar.

Prior to installation in the chamber the force transducer was calibrated with known masses in the range 50-300 g (approximately $0.5-3 \mathrm{~N}$ ). A total of 10 readings for each weight were used to construct a calibration line to check linearity over the range. The gradient of this line was then used as the calibration constant to extract force from the voltage signal recorded by LabView.

For each test condition a total of 100 injections were conducted with a rest period of $1 \mathrm{~s}$ between each injection. The recorded

Table 1

Injector nozzle diameters and $K$-factor.

\begin{tabular}{lll}
\hline$D_{\text {inlet }}(\mu \mathrm{m})$ & $D_{\text {outlet }}(\mu \mathrm{m})$ & $K$ factor $(\mu \mathrm{m})$ \\
\hline 140 & 120 & 2 \\
\hline
\end{tabular}


voltage signal was scaled to give the force using the calibration constant.

Each fuel used in the experimentation was characterized by density and viscosity. Fuel density was measured using a $10 \mathrm{ml}$ measuring cylinder and a set of scales (Ohaus Adventurer AV114). Each measurement was repeated 10 times with the same measuring cylinder. The fuel viscosities were determined using a TA Instruments AR 2000 rheometer. Shear rate was automatically adjusted by the rheometer from 10 to $1000 \mathrm{~s}^{-1}$.

To complete the hydraulic characterization, the total mass of injected fuel $(m)$ must be known. This was achieved by injecting the fuel into a glass bottle with an adapted lid. After 500 injections, the bottle was weighted using a Ohaus Adventurer AV114 scale. The mean mass of fuel per injection was then determined. This process was repeated 5 times for each condition. The instantaneous mass flow rate was determined using the mean, scaled momentum values and the total mass injected using Eq. (6).

The emulsified fuels were made using the Diesel fuel with the addition of $10 \%$ and $20 \%$ of water (by mass). Production was accomplished as follows. Approximately $500 \mathrm{ml}$ of the base fuel was weighed to determine the mass percentage of water to be added. The emulsion was stabilized with the addition $1 \%$ by total mass of surfactant. The choice of suitable surfactant was made using the hydrophilic-lipophilic balance (HLB) methodology and results of previous studies [7,26-29]. The two surfactants used were Span 80, a non-ionic lipophilic surfactant with a HLB of 4.3 and Tween 80, a non-ionic hydrophilic surfactant with a HLB of 15.

Samples were drawn from these emulsions into $10 \mathrm{ml}$ graduated flasks and left for a 5 day period to assess stability. It was found that the surfactant mix resulting in a HLB of approximately 6.4 (80\% Span 80 and $20 \%$ Tween 80 ) resulted in the most stable emulsion. It has been seen in studies elsewhere $[27,30]$ that the use of mixture of Span 80 and Tween 80 leads to better results compared with the use of a single surfactant with equivalent value of HLB.

Once the required components had been weighed the emulsion was constructed. The surfactant mixture was first dissolved in the Diesel fuel and then the water was added. The mixture was then subjected to $5000 \mathrm{~J}$ of ultrasonic mixing using an ultrasound generator (Sonics, Vibra-cell $750 \mathrm{~W}, 20 \mathrm{kHz}$ ) and a ultrasonic probe (Model CV33). The probe was inserted so that its end was at mid-depth in the mixture. Due to the heating effect that arises when a liquid is subjected to ultrasonic waves in this nature, the mixtures where kept cool during the process using an iced water bath. The ultrasonic method of mixing has been used elsewhere [3,31-33] and has been shown to result in: smaller mean droplet size; larger number of water droplets that are distributed more uniformly in the continuous phase; lower separating rates of the water droplets from the emulsions when compared with emulsions prepared by a more conventional mechanical homogenizer [3]. Ultrasonic emulsification is therefore recognized as a fast, efficient technique for producing tiny and uniformly-sized droplets.

\section{Results and discussion}

\subsection{Spray momentum flux}

The transient variation of $\dot{M}$ over the entire injection period for Diesel fuel, D10 and D20 injections for all conditions are shown in Fig. 3. For all cases here the curve shows the arithmetic mean $\dot{M}$ of 100 injections. No signal smoothing has been applied. A sampling rate of $100 \mathrm{kHz}$ has been used (sample made every $10 \mu \mathrm{s}$ ). In this plot no correction to remove any injection delay has been applied.

The injection delay is the time period between the injection being initiated using the control software (TTL signal sent to driver

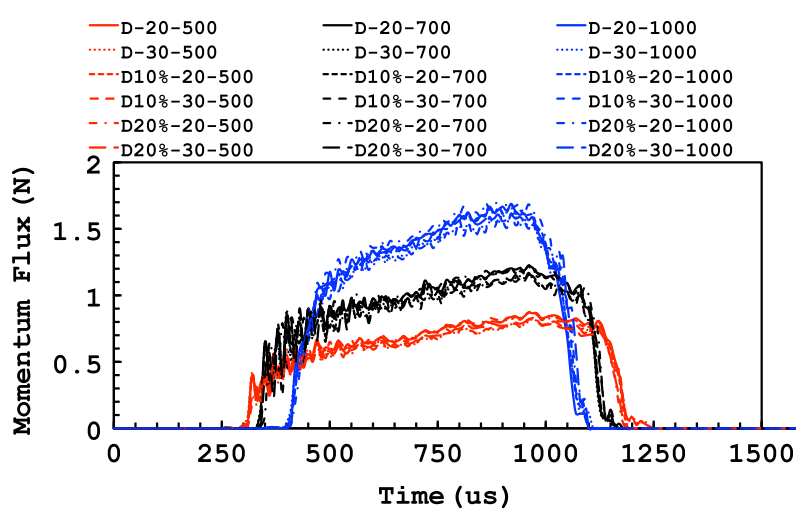

Fig. 3. Fuel spray momentum flux variation with time, after the start of data recording (with no injection delay removed) for Diesel fuel, D10 and D20. $P_{i n j}=500$ (red), 700 (black) and 1000 bar (blue) $\rho_{a}=22.6 \mathrm{~kg} / \mathrm{m}^{3}$ ( $P_{a m b}=20$ bar). $\rho_{a}=34.5 \mathrm{~kg} / \mathrm{m}^{3}$ $\left(P_{a m b}=30\right.$ bar $)$. (For interpretation of the references to colour in this figure legend, the reader is referred to the web version of this article.)

from the PC) and fuel spray starting. The delay consists of an electronic fraction from the control hardware, most notably the injector driver, and a hydraulic fraction which arises due the high pressure fuel acting upon various valves and control surfaces in the injector itself. The portion of the delay that has been termed "electronic delay" is assumed to be constant for all conditions and is ignored from here on. The variation in injection delay, shown by the variation in the time, $t$ when $\dot{M}>0$ is entirely from the variation in the "hydraulic delay". As the injection pressure is increased from 500 to 700 and 1000 bar, the injection delay increases from 290 to 320 to $390 \mu$ s respectively. The end of injection is much harder to qualify as the measured $\dot{M}$ does not drop to zero in the same manner as it increased from zero at the SOI.

As the injection pressure is increased, the EOI occurs earlier, with EOI at approximately 1190, 1130 and $1090 \mu \mathrm{s}\left(P_{i n j}=500\right.$, 700 and 1000 bar respectively). The hydraulic delay is mirrored at the EOI, leading to a decrease in the actual duration of injection with increasing injection pressure. The injection duration used here is quite short, with a TTL signal of $0.5 \mathrm{~ms}$ sent to the injector driver. The effective duration of the actual injection event is 910,810 and $700 \mu$ s for $P_{i n j}=500,700$ and 1000 bar respectively. Emulsification had no influence on the injection delay and duration.

The hydraulic delay is an effect of the design of the injector. The injector is an indirect, electro-hydraulic servo type utilizing an unbalanced control valve [36]. The piezo actuator moves a mushroom valve in the injector's control chamber. When actuated, the mushroom valve opens, allowing high pressure fuel in the control chamber to be dumped out of the fuel return port of the injector. The reduction of pressure in the control chamber allows the needle to snap up, opening the nozzle. The mushroom valve is held closed by a spring and the fuel system pressure. Any increase in injection pressure will result in a larger force keeping the mushroom valve closed, hence increasing the time it will take to open the mushroom valve when the piezo is actuated. This is noted to be a consequence of this type of injector design [37].

Figs. 4 and 5 show the transient variation of $\dot{M}$ over the entire injection period for Diesel fuel, D10 and D20 injection sprays into the ambient density, $\rho_{a}$, of $22.6 \mathrm{~kg} / \mathrm{m}^{3}$ and $34.5 \mathrm{~kg} / \mathrm{m}^{3}$ respectively. The initial non-zero $\dot{M}$ measurement is used to align the SOI for each condition so as to allow a comparison between the cases with the hydraulic delay removed. The SOI for each case has been aligned at $t=50 \mu \mathrm{s}$. For all conditions the initial increase in $\dot{M}$ is extremely rapid as the spray exits the injector nozzle and strikes the transducer target. The $\dot{M}$ increases at a higher rate during the 


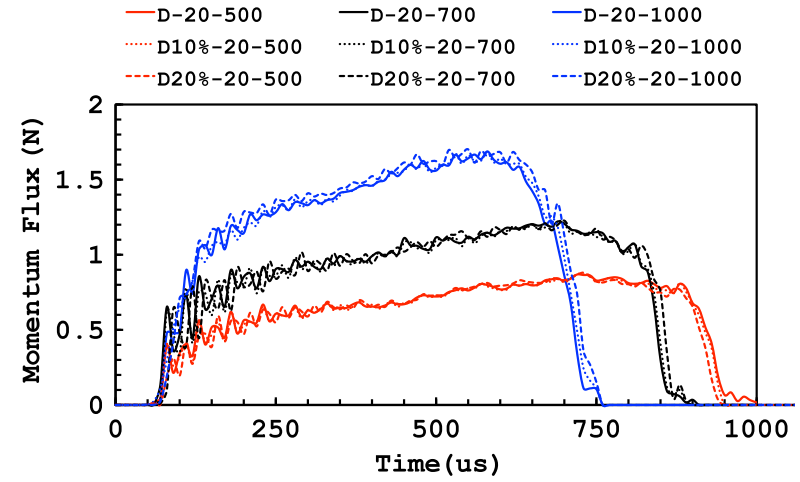

Fig. 4. Fuel spray momentum flux variation with time after SOI for Diesel fuel, D10 and D20. $P_{i n j}=500$ (red), 700 (black) and 1000 bar (blue) $\rho_{a}=22.6 \mathrm{~kg} / \mathrm{m}^{3}$ $\left(P_{a m b}=20 \mathrm{bar}\right)$. (For interpretation of the references to colour in this figure legend, the reader is referred to the web version of this article.)

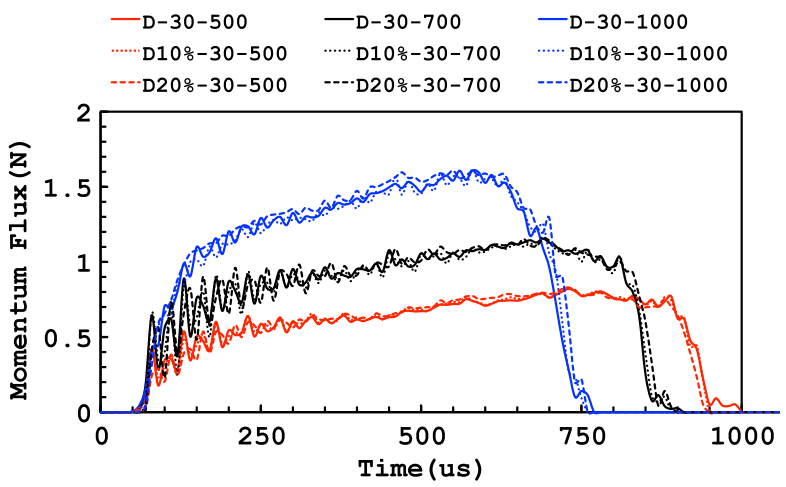

Fig. 5. Fuel spray momentum flux variation with time after SOI for Diesel fuel, D10 and D20. $P_{\text {inj }}=500$ (red), 700 (black) and 1000 bar (blue) $\rho_{a}=34.5 \mathrm{~kg} / \mathrm{m}^{3}$ $\left(P_{a m b}=30\right.$ bar). (For interpretation of the references to colour in this figure legend, the reader is referred to the web version of this article.)

early period (100-250 $\mu$ s after SOI in Figs. 4 and 5) when a higher injection pressure was applied. Throughout the injection period a higher injection pressure results in a larger $\dot{M}$. During the first $50 \mu$ s of the injection there is considerable overlap of the $\dot{M}$ curves and it is difficult to clearly state any injection pressure or ambient density effects. After approximately $100 \mu$ s the slope of the curve for all cases reduces and the variation of the momentum flux appears almost linear with time until peak momentum flux is reached. It is at around $100 \mu$ s after SOI that $\dot{M}$ of the injections into the ambient density of $22.6 \mathrm{~kg} / \mathrm{m}^{3}$ increase in size relative to the $34.5 \mathrm{~kg} / \mathrm{m}^{3}$ curves.

The mean $\dot{M}$ signal shown in the figures so far shows a large variation during the early period of the injection. This must be due to rapid transients in the spray structure which may be termed as hydraulic noise. When the curves for the same injection pressures are compared (with hydraulic delay removed, Figs. 4 and 5) there is reasonably good alignment between this hydraulic noise which suggests this noise is repeatable and a feature of the nozzle and transducer target interaction at these injection conditions ( $P_{i n j}, \rho_{a}$, fuel type, injector energizing time).

The largest 10 values around the peak $\dot{M}$ value are used to determine a mean peak value of $\dot{M}$ for each condition $\left(\dot{M}_{\text {mean }}\right)$. This mean peak value of $\dot{M}$ is a representative value for each test condition and is used to construct Fig. 6. During preliminary testing, the momentum fluxes for longer duration injections of $2 \mathrm{~ms}$ were also collected. It was observed that the using maximum 10 values for

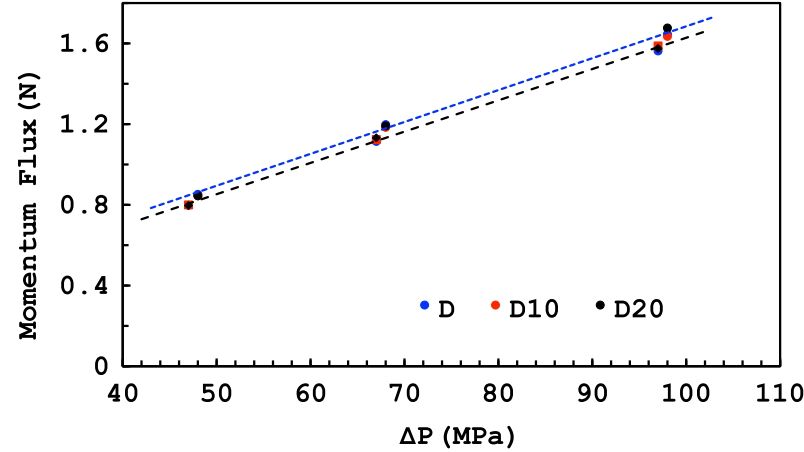

Fig. 6. Average peak value of the momentum flux for Diesel fuel (blue), D10 (red) and D20 (black) with the pressure drop across the injector nozzle $(\Delta P)$. The blue broken line is fitted to the injections with $\rho_{a}=22.6 \mathrm{~kg} / \mathrm{m}^{3}\left(P_{a m b}=20 \mathrm{bar}\right)$. The black broken line is fitted to the injections with $\rho_{a}=34.5 \mathrm{~kg} / \mathrm{m}^{3}\left(P_{a m b}=30 \mathrm{bar}\right)$. (For interpretation of the references to colour in this figure legend, the reader is referred to the web version of this article.)

the $0.5 \mathrm{~ms}$ injections is suitable as a representative value for each condition. The linear relationship between the injection pressure applied and the measured $\dot{M}$ is demonstrated, as is expected from theory. The upper, blue broken line on the plot is a linear trend line fitted to the points collected for injections into an ambient density of $22.6 \mathrm{~kg} / \mathrm{m}^{3}$. The lower, broken black line is a trend line fitted to the points collected for injections into an ambient density of $34.5 \mathrm{~kg} / \mathrm{m}^{3}$. The pressure drop across the nozzle for the different ambient densities is different, as the ambient density was changed by changing the pressure in the chamber. The theory introduced in Section 1.2 and discussed in previous work [14,34] suggests that $\dot{M}$ measured in this way would have no dependents on the density of the atmosphere into which the injection is taking place. If this was the case, and $\dot{M}$ was only dependent on the pressure drop across the nozzle, then one would expect all points to lie on the same line.

Although the effect is small, the high number of samples collected suggests that this difference is statistically significant. This is a surprise considering that $\dot{M}$ was measured $0.5 \mathrm{~mm}$ form the nozzle exit. It was not expected that the ambient density would have any effect on $\dot{M}$ at this distance from the nozzle as the spray is expected to mainly consist of liquid core at this range, which would have had little interaction with the ambient gas. The results here suggest that the liquid core of the spray is experiencing momentum exchange with the surrounding gas even at this distance from the nozzle. For each condition, the emulsification has had little, to no effect in the mean peak $\dot{M}$.

\subsection{Total mass injected}

Table 2 shows the average mass of fuel injected for each fuel at each pressure, determined from the 5 batches of 500 injections.

Table 2

Mass of fuel injected per injection event for each fuel considered.

\begin{tabular}{llll}
\hline Fuel & Injection pressure & Mass/injection $(\mathrm{g})$ & Relative $\sigma(\%)$ \\
\hline $\mathrm{D}$ & $500 \mathrm{bar}$ & 0.002353 & 0.2 \\
& $700 \mathrm{bar}$ & 0.002662 & 0.4 \\
& $1000 \mathrm{bar}$ & 0.002752 & 0.8 \\
$\mathrm{D} 10 \%$ & $500 \mathrm{bar}$ & 0.002255 & 1.0 \\
& $700 \mathrm{bar}$ & 0.002492 & 0.5 \\
& $1000 \mathrm{bar}$ & 0.002630 & 1 \\
$\mathrm{D} 20 \%$ & $500 \mathrm{bar}$ & 0.002275 & 0.5 \\
& $700 \mathrm{bar}$ & 0.002519 & 0.6 \\
& $1000 \mathrm{bar}$ & 0.002652 & 1.8 \\
\hline
\end{tabular}


The relative standard deviation is included to demonstrate the high repeatability between the 5 cases. As has been shown, the spray $\dot{M}$ has a dependence on the ambient density of the injection environment. It would be sensible to assume that the ambient density may have an effect on the mass injected which, considering all masses were obtained at atmospheric conditions, is not considered here.

The data from Table 2 has been plotted and shown in Fig. 7. The mass of Diesel fuel injected for each condition is larger than both emulsions for each injection pressure. The two emulsions show a very similar injected mass. The difference between the Diesel fuel and emulsions is almost the same at each injection pressure.

\subsection{Fuel densities and viscosity}

The density and viscosity for each fuel has also been determined and are presented in Table 3. For all cases the viscosity was observed to reach a constant when the shear rate was between 400 and $1000 \mathrm{~s}^{-1}$. The mean value presented is the determined from taking the mean value of this constant period. The measurement was repeated three times and these 3 mean values were used to obtain a mean viscosity of the fuels, stated in Pa s.

The emulsification of the fuel resulted in a small increase in the fuel density. This is expected due to the addition of water which has a higher density than the neat Diesel. The measured density has been compared to the density obtained from the model of an ideal mixture. The ideal mixture may be defined according to two different model mixtures. One relies on the assumption that the volume of the solution is proportional to the mass and uses the mass fraction of each substance and their densities.

$\rho_{n}=\sum\left(x_{i} \rho_{i}\right) n$

where $x_{i}$ is the mass fraction. The second method assumes the volumes are additive and may be more appropriate for immiscible liquids such as water and Diesel fuel.

$1 / \rho_{n}=\sum\left(x_{i} / \rho_{i}\right) n$

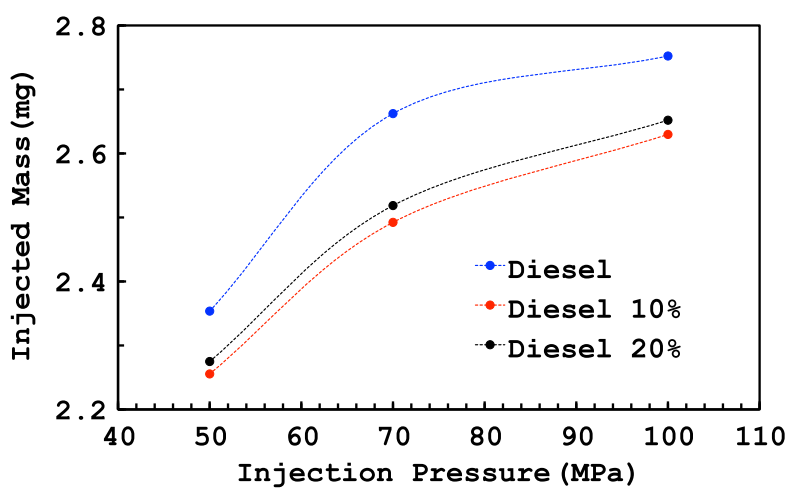

Fig. 7. Total mass of fuel injected for the Diesel (blue), D10 (red) and D20 (black) fuels at $P_{i n j}=500,700$ and 1000 bar (50,70 and $100 \mathrm{MPa}$ ). (For interpretation of the references to colour in this figure legend, the reader is referred to the web version of this article.)

Table 3

Fuel density and viscosity.

\begin{tabular}{lll}
\hline Fuel & Density $\left(\mathrm{kg} / \mathrm{m}^{3}\right)$ & Viscosity (Pa s) \\
\hline Diesel & 814 & 0.00588 \\
Diesel 10/\% water & 829 & 0.00669 \\
Diesel 20/\% water & 876 & 0.00678 \\
\hline
\end{tabular}

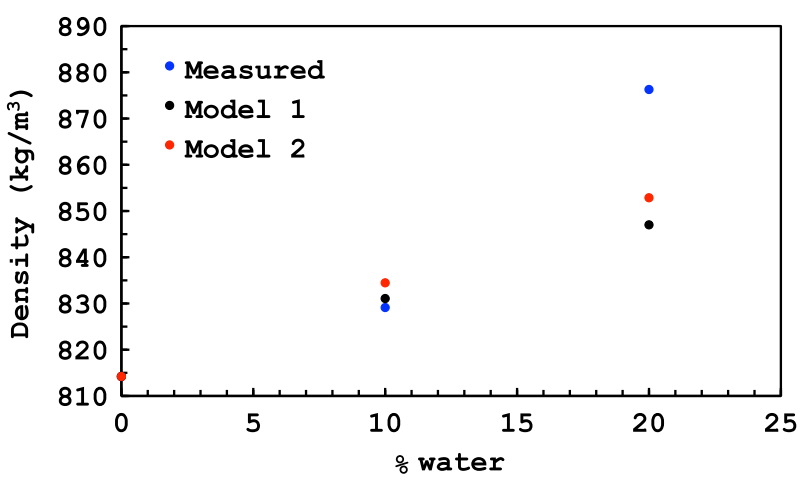

Fig. 8. Density of the fuels with percentage of water added. Density determined form measurement in blue, density calculated using Eq. (10) in black. Density calculated using Eq. (11) in red. (For interpretation of the references to colour in this figure legend, the reader is referred to the web version of this article.)

The results of the measured and the two predictive models are shown in Fig. 8. The measured density for the D10 fuel is similar to the two predictive models. The measured density for the D20 fuel is much larger than is predicted by the two mixing models. This suggests that for the emulsified fuel, as the water content increases, the surfactant is acting to reduce the volume that the water would take up in a simple mixture. The emulsification process is not a simple mixing process and results in a complex mixture of dispersed droplets with a range of sizes and surface areas. It is evident that as the water is added, the dispersed phase becomes much larger and the droplets may be able to compact more closely together leading to an increase in the density away from a simple linear mixing model.

The small increase in density of the emulsified fuels would be expected to lead to an increase in the injected mass, considering the injected mass is given by $A_{\text {geo }} \sqrt{\rho_{f} 2 \Delta P}$. This is not what was observed (Fig. 7). The emulsification process has resulted in an increase in the measured viscosity compared to neat Diesel. This increase in viscosity must be countering the increase in fuel density to reduce the mass of fuel that is injected during the needle opening time.

\subsection{Instantaneous mass flow rate}

Using Eq. (6), the transient $\dot{M}$ and the independently measured total mass of injected fuel the transient instantaneous mass flow rate, $\dot{m}$, for each condition has been determined. The integral of the square root of the momentum flux (see Eq. (6)) is defined by the area below the $\dot{M}$ curve and has been calculated numerically using the trapezium technique in Matlab. An example of the transient $\dot{m}$ profiles are shown in Fig. 9. This example includes profiles for each fuel at each injection pressure with the ambient density of $22.6 \mathrm{~kg} / \mathrm{m}^{3}$.

The $\dot{M}$ profiles and the $\dot{m}$ profiles are essentially the same shape with scaling between the two proportional to the spray velocity (based upon assumptions pertaining to Eq. (1)). Both profiles may be used in a modeling process to elucidate temporal evolution of fuel concentration in the combustion chamber and may also be used in the development of injector profile shaping for improved fuel metering and combustion characteristics. The total mass injected is different for each fuel, which leads to a change in $\dot{m}$ for the emulsified fuels when compared with the Diesel fuel sprays. The 10 largest values of $\dot{m}$ for each condition have been used to produce a mean peak value in a similar manner as was done for $\dot{M}$ mean peak values in Fig. 6 . 


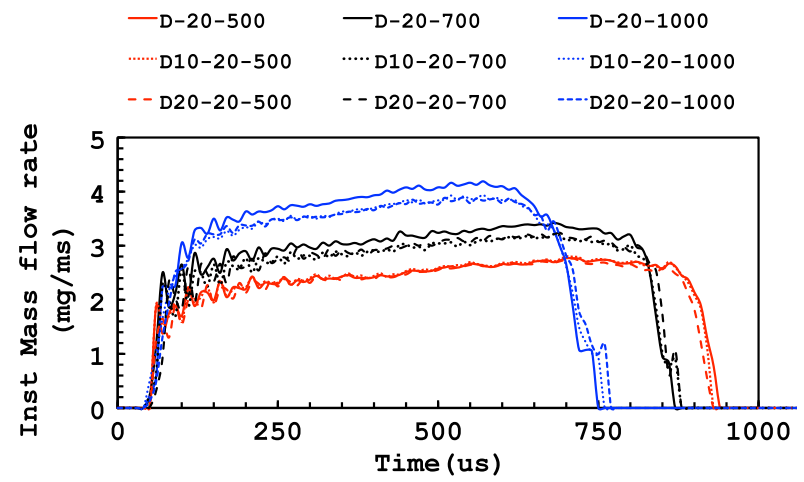

Fig. 9. Instantaneous mass flow rate profile for Diesel fuel (solid line), D10 (fine broken line) and D20 (course broken line). $P_{\text {inj }}=500$ (red), 700 (black) and 1000 bar (blue) $\rho_{a}=22.6 \mathrm{~kg} / \mathrm{m}^{3} \quad\left(P_{a m b}=20 \mathrm{bar}\right)$. (For interpretation of the references to colour in this figure legend, the reader is referred to the web version of this article.)

The mean peak values have been used as a representative value of $\dot{m}$ for each condition and have been used to produce Fig. 10. The linear dependence of $\dot{m}$ with the square root of the pressure drop across the nozzle is demonstrated. The two emulsified fuels have a near identical peak $\dot{m}$ at each injection pressure. As the injection pressure is increased the difference between the neat Diesel and the emulsified fuels gets larger. The $\dot{m}$ mean peak value is almost identical at 500 bar injection pressure for each fuel. At 1000 bar injection pressure there is a difference in $\dot{m}$ of approximately $0.3 \mathrm{mg} / \mathrm{ms}$. The difference is small but this reflects the short time scale and mass scales that are involved in the measurement of these injection events. The difference in the instantaneous mass flow rates between the Diesel fuel and the emulsified fuels is to be expected when the difference in the total mass injected is examined in Fig. 7. The technique may be considered successful at producing an instantaneous mass rate profile that may be used as an input into multidimensional engine models.

\subsection{Discharge coefficient}

The discharge coefficient has been determined using Eq. (7) with: $\dot{m}$; the geometrical cross sectional area $\left(A_{g e o}\right)$ of the nozzle orifice, determined using the silicone mold of the nozzle; the fuel density and; the injection pressure.

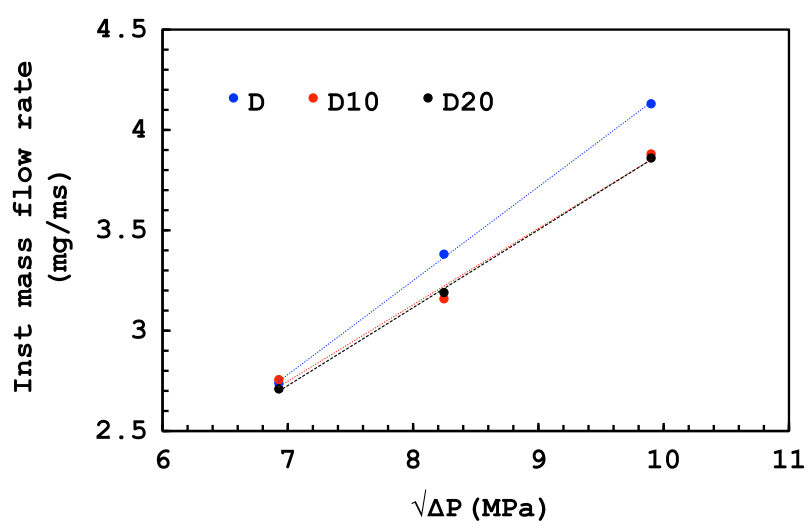

Fig. 10. Mean peak value of the instantaneous mass flow rate for Diesle fuel (blue), D10 (red) and D20 (black) with the square root of the pressure drop across the injector nozzle $(\sqrt{\Delta P}) . \rho_{a}=22.6 \mathrm{~kg} / \mathrm{m}^{3}\left(P_{a m b}=20 \mathrm{bar}\right)$. (For interpretation of the references to colour in this figure legend, the reader is referred to the web version of this article.)

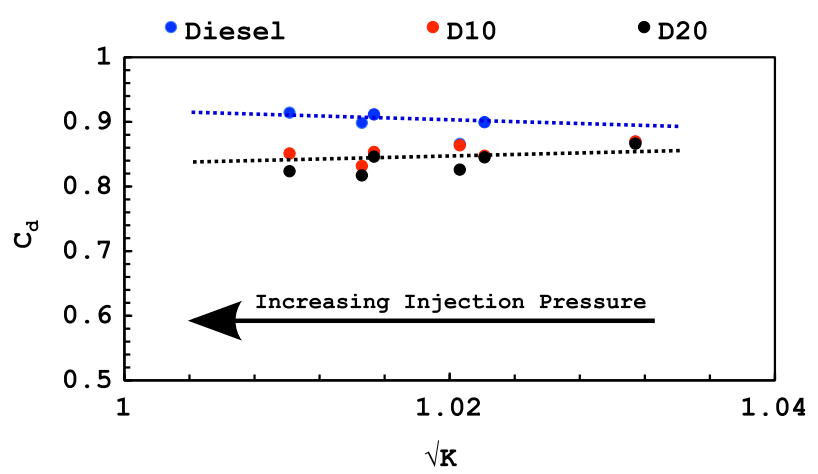

Fig. 11. Nozzle discharge coefficient with the square root of the cavitation number (Eq. (8)) for Diesel fuel (blue), D10 (red) and D20 (black). Trend line has been fitted separately to Diesel fuel (red) and the emulsions (blue). (For interpretation of the references to colour in this figure legend, the reader is referred to the web version of this article.)

Fig. 11 shows the $C_{d}$ for each condition with the square root of the cavitation number. An increase in injection pressure results in a decrease in the cavitation number hence the arrow included in Fig. 11 is in the direction of increasing injection pressure. The emulsification has lead to a reduction in the nozzle discharge coefficient compared with the neat Diesel conditions. It is evident that $C_{d}$ is not really changing with the change in injection pressure (hence $\sqrt{K}$ ) and there is no evidence that the nozzle is cavitating for any conditions or that $K_{\text {crit }}$ has been observed.

The $K$-factor (Table 1 ) and the inlet radius have effectively suppressed cavitation in the nozzle at the conditions considered here. It is observed that the discharge coefficient varies little with the change in $K$ for all fuels. The emulsification process, though reducing the discharge coefficient, has not induced any cavitation at these injection conditions. At the injection pressure of $1000 \mathrm{bar}$ (the lower $\sqrt{K}$ ) the difference in $C_{d}$ between the Diesel fuel and the emulsions is significant. The range of $K$ values examined in this study is limited when compared with other work which use this analysis $[15,19]$ so the conditions where cavitation may start cannot be assessed. Theory states that under these non-cavitating conditions, the flow is dependent on the Reynolds number [19]. To examine the Reynolds number, the flow mean velocity is required along with the fluid density and viscosity. The characteristic length scale applied is the nozzle mean diameter, determined from the measurements in Table 1 to be $130 \mu$ s. The mean injection velocity is calculated using the mean peak momentum flux value and the mean peak instantaneous mass flow rate value in the form

$V_{\text {eff }, \text { mean }}=\frac{\dot{M}_{\text {mean }}}{\dot{m}_{\text {mean }}}$

and has been used to produced Fig. 12 .

The momentum flux measured was almost the same at each condition for each fuel. The mass flow rate for the emulsions was reduced compared with the neat Diesel. For the momentum to have been unchanged the velocity of the emulsions must be higher. This is reflected in Fig. 12 which shows that as the injection pressure was increased, the mean injection velocity of the emulsions was higher compared with the neat Diesel fuel. For each condition, the injection velocity with the ambient density of $22.6 \mathrm{~kg} / \mathrm{m}^{3}$ was higher than when the ambient density was $34.5 \mathrm{~kg} / \mathrm{m}^{3}$. According to the Reynolds number equation, Eq. (13), the higher velocity and larger density of the emulsified fuels will lead to an increase in the Reynolds number, whilst the increase in viscosity of the emulsions will results in a reduction in the Reynolds number.

$R e_{l}=\frac{v D \rho_{l}}{\mu_{l}}$ 


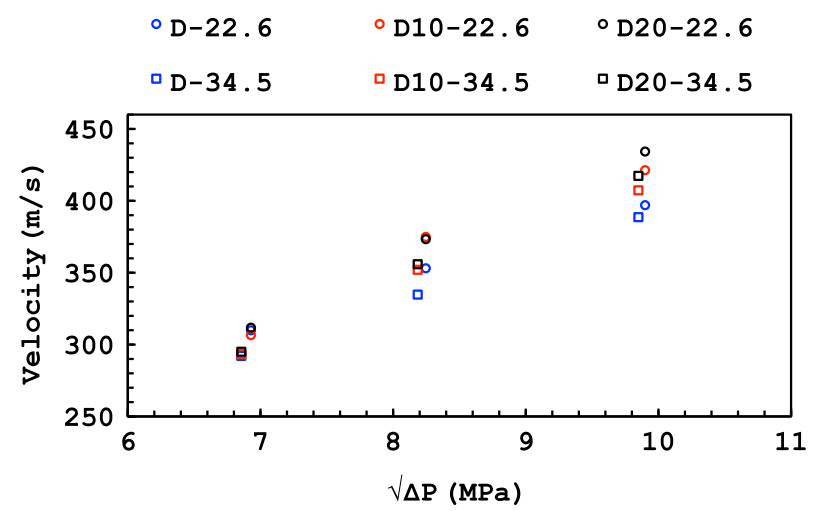

Fig. 12. Mean peak value of the injection velocity for Diesel fuel (blue), D10 (red) and D20 (black) with the square root of the pressure drop across the injector nozzle $(\sqrt{\Delta P}) . \rho_{a}=22.6 \mathrm{~kg} / \mathrm{m}^{3}\left(P_{a m b}=20\right.$ bar $)$ shown with empty circles. $\rho_{a}=34.5 \mathrm{~kg} / \mathrm{m}^{3}$ $\left(P_{a m b}=30\right.$ bar) shown with empty squares. (For interpretation of the references to colour in this figure legend, the reader is referred to the web version of this article.)

The Reynolds number of the fuel sprays here fall within the range 4700-7400. Considering the large scale over which the Reynolds number is measured, the difference in Reynolds number between the fuels is small. This suggests that, although there is no cavitation, it is may not be the Reynolds number of the flow in the nozzle which is reducing the discharge coefficient for the emulsified fuels. It is worth noting that the Reynolds numbers calculated here are a lot lower than those in previous works. This is believed to be due to the quite high viscosity measured here when compared with values of dynamic viscosity in the range of 0.002 Pa s $[15,35]$ which are often used.

\subsection{Momentum coefficient}

Whilst the discharge coefficient may be important from a fuel metering point of view, the momentum of the spray represents the energy available to the spray as it mixes and exchanges momentum with the surrounding gas. Two new coefficients are introduced based upon the momentum. One of these, the so called "momentum coefficient" [15], $C_{M}$, is analogous to the discharge coefficient, defined as the measured momentum divided by the theoretical momentum of the spray, given by

$C_{\text {Mmean }}=\frac{\dot{M}_{\text {mean }}}{A_{\text {geo }} \rho_{f} v_{\text {eff }}^{2}}=\frac{\dot{M}_{\text {mean }}}{2 A_{\text {geo }} \Delta P}$

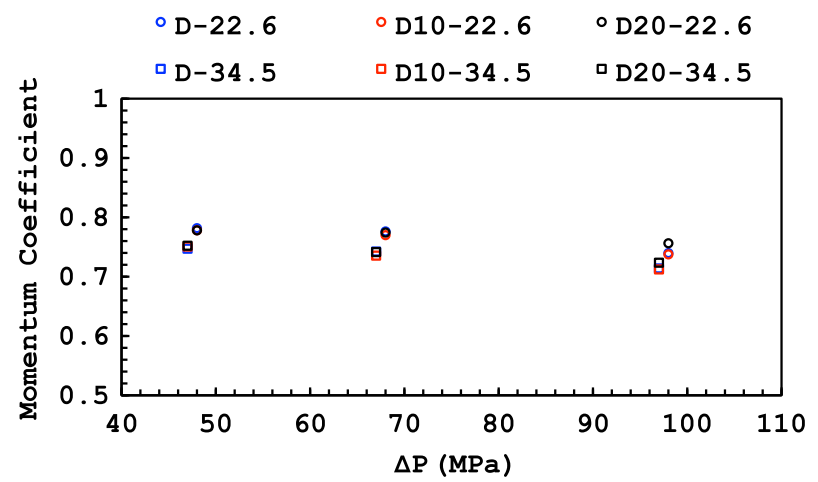

Fig. 13. Momentum coefficient (Eq. (14) of sprays for Diesel due (blue), D10 (red) and D20 (black) with the pressure drop across the injector nozzle $(\sqrt{\Delta P})$. $\rho_{a}=22.6 \mathrm{~kg} / \mathrm{m}^{3} \quad\left(P_{a m b}=20 \mathrm{bar}\right)$ shown with empty circles. $\rho_{a}=34.5 \mathrm{~kg} / \mathrm{m}^{3}$ $\left(P_{a m b}=30\right.$ bar) shown with empty squares. (For interpretation of the references to colour in this figure legend, the reader is referred to the web version of this article.)
The representative mean peak values of the momentum flux determined in Section 3.1 are used to determine the momentum coefficient. The change in the $C_{\text {Mmean }}$ with the pressure drop $(\Delta P)$ across the nozzle is shown in Fig. 13. The theoretical momentum flux of the spray increases with increasing $\Delta P$, the results shown in Fig. 6 agree with this statement, but it is only after the momentum coefficient is determined that it is clear that, in terms of momentum, the nozzle is not performing as would be expected with increasing injection pressure. For all fuels, the increase in the ambient density in the chamber has resulted in a reduction in the momentum coefficient. The emulsification has had no discernible effect on the momentum coefficient, with each fuel, at each injection condition having a very similar, almost identical momentum coefficient.

The momentum coefficient has been calculated using the theoretical momentum flux for each condition given by $2 A_{\text {geo }} \Delta P$. The geometric area of the nozzle orifice is being utilized again (which is based upon the assumptions already outlined) and may introduce a misunderstanding of the effects upon the spray momentum. The instantaneous mass flow rate has been determined using a form of normalized momentum flux measurement and the total mass injected. Any change in the cross sectional area of the fluid flow through the nozzle away from $A_{\text {geo }}$ has been accounted for in this measurement. It would seem suitable then to use the calculated mass flow rate with

$\dot{M}=\dot{m} v_{B}$

where $v_{B}$ is a theoretical velocity from Bernoulli's equation in the form

$\eta_{m}=\frac{\dot{M}}{\dot{m} \cdot \sqrt{\frac{2 \Delta P}{\rho_{f}}}}$

to give the momentum efficiency.

This separates out any area contraction effects due to cavitation and considers the losses in the nozzle orifice due to velocity changes only. The momentum efficiency for the nozzle for each fuel is shown in Fig. 14. The neat Diesel fuel sprays show a near linear decrease of the momentum efficiency with increasing injection pressure which is greater than the decrease for emulsified fuels. For each fuel at each injection pressure the higher chamber density leads to a reduction in the momentum efficiency. The D20 fuel sprays have the highest momentum efficiency at each condition, followed by the D10 with the Diesel fuel exhibiting a lower efficiency at each condition. The emulsification process has been shown to lead to an increase in the injection velocity compared with an equivalent Diesel fuel spray. This is counter the increase

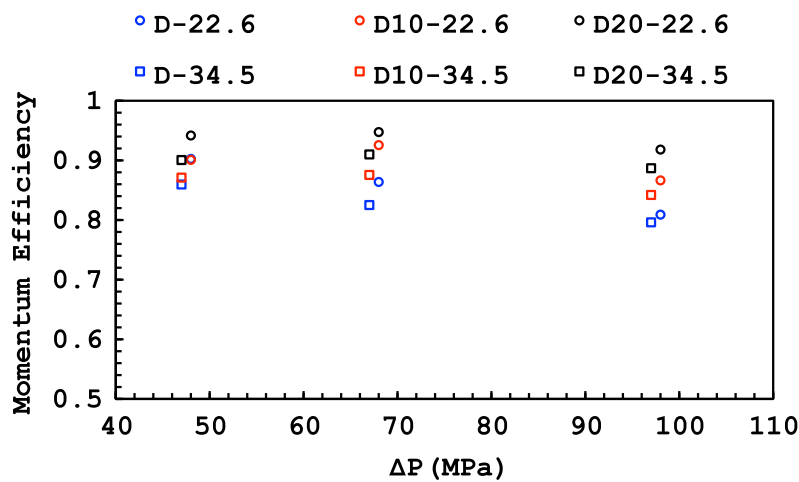

Fig. 14. Momentum efficiency (Eq. (16)) of sprays for Diesel due (blue), D10 (red) and D20 (black) with the pressure drop across the injector nozzle $(\sqrt{\Delta P})$. $\rho_{a}=22.6 \mathrm{~kg} / \mathrm{m}^{3} \quad\left(P_{a m b}=20 \mathrm{bar}\right)$ shown with empty circles. $\rho_{a}=34.5 \mathrm{~kg} / \mathrm{m}^{3}$ $\left(P_{a m b}=30\right.$ bar) shown with empty squares. (For interpretation of the references to colour in this figure legend, the reader is referred to the web version of this article.) 
in viscosity that arises due to the emulsification process, which may have been expected to reduce flow velocity.

The complex nature of the multi-component flow is not readily explained with the measurements here. It is apparent that the high viscosity, which would be expected to reduce flow velocity is not the only mechanism working on the emulsified fluid as it is injected. It is unclear what happens to the emulsion's density and viscosity as it is subjected to the high pressures of injection. The large pressure drop, coupled with the drastic variation in flow direction as the fuel flows through the injector may result in a "splitting" of the emulsion or the formation of a lower viscosity region in the nozzle through which the fuel may flow with a higher velocity.

\subsection{Summary and conclusion}

The momentum flux of Diesel fuel sprays; Diesel and 10\% water emulsion and Diesel and 20\% water have been measured using a force transducer placed at a very close distance $(0.5 \mathrm{~mm})$ from the nozzle orifice exit. The injections have been carried out with injection pressures 500, 700 and 1000 bar into a high pressure chamber filled with nitrogen with two back pressures, 20 and $30 \mathrm{bar}$, resulting in ambient densities of $22.6 \mathrm{~kg} / \mathrm{m}^{3}$ and $34.5 \mathrm{~kg} /$ $\mathrm{m}^{3}$. Spray duration was short at $500 \mu$ s but it has been demonstrated that in this time the spray reached a reasonable representative momentum flux value for each condition. The 10 largest readings have been used to produce a mean peak value that is a single value from the transient region which is representative of the momentum flux for each condition.

- Increasing the injection pressure resulted in an extended hydraulic delay in the injector and earlier closing time of the injector.

- Increasing ambient density in the chamber results in a decrease in the measured momentum flux, greater than would be expected due to the back pressure change that accompanies an ambient density change. The spray had not emerged very far into the chamber $(0.5 \mathrm{~mm})$ before the ambient density has influenced the spray.

- The emulsified fuels had a very similar, almost identical momentum flux as the neat Diesel fuel. The emulsification has not had any effect on the injection delay period or the injection closing time.

- Fuel density and viscosity has been measured. The emulsions have a larger density than the neat Diesel fuel and a higher viscosity.

- The total mass of fuel injected during an injection event, for each fuel, at the three injection pressures considered was measured. The larger density of the emulsions has not been reflected in the total mass injected, with the total mass of fuel injected for the emulsified fuels less than for neat Diesel at each injection pressure.

- Instantaneous mass flow rate has been determined using a normalized form of the momentum flux measurement and the independently measured total mass injected. Instantaneous mass flow shown a linear increase with $\sqrt{\Delta P}$.

- The nozzle discharge coefficient has been determined and examined with the cavitation number. The emulsions tend to have a lower discharge coefficient. There is no evidence that the nozzle is cavitating for any of the fuels at the conditions tested. It is believed the nozzle design has effectively suppressed cavitation.

- Injection velocity has been calculated using the mean peak momentum values and the mean peak mass flow values. The emulsified fuels have tended to have a high injection velocity than the neat Diesel fuel sprays. The velocity, along with density and viscosity has been used to determine the Reynolds number for each case. The Reynolds number has shown little variation between the fuels at each condition. It is concluded that there is another mechanism resulting in the lower discharge coefficient and high injection velocities observed with the emulsified fuels.

- The momentum coefficient has been determined for the sprays and is analogous to the discharge coefficient. The emulsification has little to no effect on the momentum coefficient. It is evident that the increase in ambient density reduces the momentum coefficient for each case.

- The momentum efficiency is introduced, which uses the instantaneous mass measurement and the theoretical velocity of the spray. The emulsified fuels have a larger momentum efficiency, a result of their high injection velocity compared with the neat Diesel fuel.

\section{Acknowledgements}

The authors wish to thank Advanced Engine Research, Basildon, UK for their continued financial and technical support throughout this project. Principal investigator Professor T. Korakianitis

\section{References}

[1] Fahd ME Alam, Wenming Y, Lee PS, Chou SK, Yap CR. Experimental investigation of the performance and emission characteristics of direct injection diesel engine by water emulsion diesel under varying engine load condition. Appl Energy 2013;102(0):1042-9.

[2] Rosen MJ. Surfactants and interfacial phenomena. 2nd ed. New York, NY: Wiley; 1989.

[3] Lin C Yuan, Chen L. Comparison of fuel properties and emission characteristics of two- and three-phase emulsions prepared by ultrasonically vibrating and mechanically homogenizing emulsification methods. Fuel 2008;87(1011):2154-61.

[4] Armas O, Ballesteros R, Martos FJ, Agudelo JR. Characterization of light duty diesel engine pollutant emissions using water-emulsified fuel. Fuel 2005;84 (7-8):1011-8.

[5] Qi DH, Chen H, Matthews RD, Bian YZH. Combustion and emission characteristics of ethanol, biodiesel with water micro-emulsions used in a direct injection compression ignition engine. Fuel 2010;89(5):958-64.

[6] Morozumi Y, Saito Y. Effect of physical properties on microexplosion occurrence in water-in-oil emulsion droplets. Energy Fuels 2010;24 (3):1854-9.

[7] Huo M, Lin S, Nithyanandan K, Liu H, Lee CF. Effects of injection pressure and ambient temperature on spray characteristics of water emulsified diesel. In: ILASS Americas, 24th annual conference on liquid atomization and spray systems; 2012.

[8] Ghojel JI, Tran X Thien. Ignition characteristics of diesel water emulsion sprays in a constant-volume vessel: effect of injection pressure and water content. Energy Fuels 2010;24(7):3860-6.

[9] Postrioti L, Buitoni G, Pesce FC, Ciaravino C. Zeuch method-based injection rate analysis of a common-rail system operated with advanced injection strategies. Fuel 2014;128(0):188-98.

[10] Naber J, Siebers D. Effects of gas density and vaporization on penetration and dispersion of diesel sprays. SAE technical paper, 960034; 1996.

[11] Husberg T, Manente V, Ehleskog R, Andersson S. Fuel flow impingement measurements on multi-orifice diesel nozzles. SAE technical paper, 2006-011552; 2006.

[12] Lindstrom M, Angstrom H. Development of a fuel spray impulse measurement device and correlation with time resolved mass flow. SAE technical paper, 2009-01-1880; 2009.

[13] Ganippa L, Andersson S, Chomiak J. Transient measurements of discharge coefficients of diesel nozzles. SAE technical paper, 2000-01-2788; 2000.

[14] Sangiah DK, Ganippa LC. Application of spray impingement technique for characterization of high pressure sprays from multi-hole diesel nozzles. Int J Therm Sci 2010;49(2):409-17.

[15] Payri R, Salvador FJ, Gimeno J, Soare V. Determination of diesel sprays characteristics in real engine in-cylinder air density and pressure conditions. J Mech Sci Technol 2005;19(11):2040-52.

[16] Johnson SE, Nesbitt JE, Naber JD. Mass and momentum flux measurements with a high pressure common rail diesel fuel injector. In: ASME 2010 international combustion engine division fall technical conference; 2010.

[17] Kempenaar JG, Mueller CJ, Shollenberger KA, Lakshminarasimhan Krishna. An instrument for measuring orifice-specific fuel-injection rate from a multiorifice nozzle. In: ASME 2008 fluids engineering conferences. Paper No. FEDSM2008-55047:305-312; 2008. 
[18] Payri F, Bermudez V, Payri R, Salvador FJ. The influence of cavitation on the internal flow and the spray characteristics in diesel injection nozzles. Fuel 2004;83:419-31.

[19] Desantes JM, Payri R, Salvador FJ, Gimeno J. Measurements of spray momentum for the study of cavitation in diesel injection nozzles. SAE technical paper, 2003-01-0703; 2003.

[20] Postrioti L, Mariani F, Battistoni M. Experimental and numerica momentum flux evaluation of high pressure diesel spray. Fuel 2012;98(0): 149-63.

[21] Postrioti L, Battistoni M. Evaluation of diesel spray momentum flux in transient flow conditions. SAE technical paper, 2010-01-2244; 2010.

[22] Postrioti L, Battistoni M. Analysis of diesel spray momentum flux spatial distribution. SAE technical paper, 2011-01-0682; 2011.

[23] Postrioti L, Bosi M. Momentum flux spatial distribution and PDS analysis of a GDI spray. SAE technical paper, 2012-01-0459; 2012.

[24] Emberson D. Diesel fuel and diesel fuel with water emulsions spray and combustion characterization. PhD thesis, QMUL; 2015.

[25] Luo F, Cui Hg, Dong S. Transient measuring method for injection rate of each nozzle hole based on spray momentum flux. Fuel 2014:125(0):20-9.

[26] Abu-Zaid M. Performance of single cylinder, direct injection diesel engine using water fuel emulsions. Energy Convers Manage 2004;45(5): 697-705.

[27] Lin YS, Lin HP. Spray characteristics of emulsified castor biodiesel on engine emissions and deposit formation. Renew Energy 2011;36(12):3507-16.
[28] Maiboom A, Tauzia X. NOx and PM emissions reduction on an automotive HSDI diesel engine with water-in-diesel emulsion and EGR: an experimental study. Fuel 2011;90(11):3179-92.

[29] Zhang D, Lin Y, Li A, Tarasov VV. Emulsification for castor biomass oil. Front Chem Sci Eng 2011;5(1):96-101.

[30] Attia AMA, Kulchitskiy AR. Influence of the structure of water-in-fuel emulsion on diesel engine performance. Fuel 2014;116(0):703-8.

[31] Bulent Koc A, Abdullah M. Performance and NOx emissions of a diesel engine fueled with biodiesel-diesel-water nanoemulsions. Fuel Process Technol 2013;109(0):70-7.

[32] Cucheval A, Chow RCY. A study on the emulsification of oil by power ultrasound. Ultrason Sonochem 2008;15(5):916-20.

[33] Lin CY, Chen LW. Emulsification characteristics of three- and two-phase emulsions prepared by the ultrasonic emulsification method. Fuel Process Technol 2006;87(4):309-17.

[34] Dernotte J, Foucher F, Hespel C, Houill S. Experimental study of the effect of fuel properties on the diesel injection process. In: Proceedings of the European combustion meeting 2011; 2011.

[35] Benajes J, Pastor JV, Payri R, Plazas AH. Analysis of the influence of diesel nozzle geometry in the injection rate characteristic. J Fluids Eng 2004;126 (1):63-71.

[36] Siemens. Siemens VDO automotive lynx PCR system; 2004

[37] Lai MCD, Dingle PJ. Diesel common rail and advanced fuel injection systems. SAE international; 2005. 